\title{
Changes of 5-hydroxymethylcytosine distribution during myeloid and lymphoid differentiation of CD34+ cells
}

\author{
Xavier Tekpli,11, Alfonso Urbanucci 1,2, Adnan Hashim ${ }^{1}$, Cathrine B. Vågbø ${ }^{3}$, Robert Lyle ${ }^{4}$, Marianne K. Kringen" \\ Anne Cathrine Staff ${ }^{6}$, Ingunn Dybedal ${ }^{7}$, Ian G. Mills ${ }^{1,2,8}$, Arne Klungland ${ }^{9,10}$ and Judith Staerk $1,7,10^{*}$
}

\begin{abstract}
Background: Hematopoietic stem cell renewal and differentiation are regulated through epigenetic processes. The conversion of 5-methylcytosine into 5-hydroxymethylcytosine $(5 \mathrm{hmC})$ by ten-eleven-translocation enzymes provides new insights into the epigenetic regulation of gene expression during development. Here, we studied the potential gene regulatory role of $5 \mathrm{hmC}$ during human hematopoiesis.

Results: We used reduced representation of 5-hydroxymethylcytosine profiling (RRHP) to characterize $5 \mathrm{hmC}$ distribution in CD34+ cells, CD4+ T cells, CD19+ B cells, CD14+ monocytes and granulocytes. In all analyzed blood cell types, the presence of $5 \mathrm{hmC}$ at gene bodies correlates positively with gene expression, and highest $5 \mathrm{hmC}$ levels are found around transcription start sites of highly expressed genes. In CD34+ cells, $5 \mathrm{hmC}$ primes for the expression of genes regulating myeloid and lymphoid lineage commitment. Throughout blood cell differentiation, intragenic 5hmC is maintained at genes that are highly expressed and required for acquisition of the mature blood cell phenotype. Moreover, in CD34+ cells, the presence of $5 \mathrm{hmC}$ at enhancers associates with increased binding of RUNX1 and FLI1, transcription factors essential for hematopoiesis.
\end{abstract}

Conclusions: Our study provides a comprehensive genome-wide overview of $5 \mathrm{hmC}$ distribution in human hematopoietic cells and new insights into the epigenetic regulation of gene expression during human hematopoiesis.

Keywords: 5-Hydroxymethylcytosine, Epigenetics, Hematopoiesis, RUNX, FLI1

\section{Background}

Epigenetic regulation of gene expression plays an important role during stem cell renewal and cell fate determination [1]. Ten-eleven-translocation (TET) DNA dioxygenases catalyze the hydroxylation of 5-methylcytosine $(5 \mathrm{mC})$ into $5 \mathrm{hmC}$ [2]. Apart from being an intermediate during the DNA demethylation process, $5 \mathrm{hmC}$ may function as a stable epigenetic mark with regulatory function [3], a notion supported by studies showing that TET enzymes and $5 \mathrm{hmC}$ are important for early embryonic development $[2,4]$.

\footnotetext{
*Correspondence: judith.staerk@ncmm.uio.no

${ }^{1}$ Nordic European Molecular Laboratory (EMBL) Partnership, Centre

for Molecular Medicine Norway, University of Oslo, Blindern, P.O. Box 1137,

0318 Oslo, Norway

Full list of author information is available at the end of the article
}

Hematopoiesis, the lifelong formation of blood cells, is ensured by the renewal of hematopoietic stem cells and their differentiation into mature cell types. Mice lacking TET2 are characterized by pleiotropic alterations of progenitor and mature hematopoietic cells, indicating that TET2 is an important regulator of murine blood cell development [5,6]. Moreover, inactivating mutations of TET2 are found in various human blood disorders [7]. Recent studies mapping hydroxymethylated regions in blood cells indicated a role of $5 \mathrm{hmC}$ during erythropoiesis $[8]$ as well as $\mathrm{T}$ cell and $\mathrm{B}$ cell differentiation $[9,10]$. However, these reports used enrichment-based methods to detect $5 \mathrm{hmC}$ and focused on a specific lineage.

To systematically study the role of $5 \mathrm{hmC}$ during human hematopoiesis and assess the possible gene regulatory role of $5 \mathrm{hmC}$ during lymphoid and myeloid 
differentiation, we used a single-base resolution method, reduced representation of 5-hydroxymethylcytosine profiling (RRHP) [11]. We mapped $5 \mathrm{hmC}$ in CD34+ and mature peripheral blood cells $(\mathrm{CD} 4+\mathrm{T}$ cells, CD19+ B cells, CD14+ monocytes and granulocytes). By integrating $5 \mathrm{hmC}$ profiles with gene expression analysis, histone modifications as well as transcription factor (TF) binding profiles we provide molecular evidence that $5 \mathrm{hmC}$ promotes the expression of key genes important for the differentiation of CD34+ cells into either lymphoid or myeloid lineage.

\section{Results}

\section{Genome-wide distribution and quantification of $5 \mathrm{hmC}$ in hematopoietic cells}

We used RRHP analysis to quantify and assess the distribution of 5hmC-positive CCGG sites in hESC $(n=2)$, umbilical cord blood $(n=2)$ and bone marrow $(n=1)$ CD34+ cells (CB-CD34+ and BM-CD34+), as well as CD4+ T cells, CD19+ B cells, CD14+ monocytes $(n=1$ for each) and granulocytes $(n=2)$ (Fig. 1a; Additional file 1: Table S1). We found a strong correlation in replicated samples (hESC, CB-CD34+ and granulocytes) with Pearson's correlation $r>0.75$ and $p<0.05$ (Additional file 1: Table S1). We first compared $5 \mathrm{hmC}$ quantification in the different blood cell types using RRHP (Fig. 1a) and liquid chromatography tandem mass spectrometry (LC/ MS/MS) (Fig. 1b). We included human embryonic stem cells (hESC) as reference cell type in our analysis since $5 \mathrm{hmC}$ has been well characterized in those cells and it is known that pluripotent cells contain high levels of $5 \mathrm{hmC}$ $[12,13]$.

Both methods, RRHP and LC/MS/MS, showed that higher $5 \mathrm{hmC}$ levels were found in pluripotent (hESC) cells and multipotent (CD34+) cells compared to mature blood cell types (Fig. 1a, b). This was in contrast to $5 \mathrm{mC}$ levels measured by LC/MS/MS, which did not change between the different cell types (Additional file 2: Fig. $\mathrm{S} 1$ ). We next characterized the distribution of $5 \mathrm{hmC}$ in annotated elements in the different blood cell types and found that $78-82 \%$ of $5 \mathrm{hmC}$ sites localized in introns and intergenic regions (Fig. 1c). Since introns and intergenic regions represent $80 \%$ of the human genome, it was expected that most of the $5 \mathrm{hmC}$ sites will fall within these genomic elements. We therefore determined the relative density of $5 \mathrm{hmC}$ sites in annotated genomic elements (Fig. 1d) and found high $5 \mathrm{hmC}$ density in exons and promoter, indicating that the presence of $5 \mathrm{hmC}$ is particularly abundant around annotated genes. Notably, $\mathrm{T}$ lymphocytes showed higher $5 \mathrm{hmC}$ density in promoters than other cell types, possibly reflecting an important role for $5 \mathrm{hmC}$ at promoters in $\mathrm{T}$ cells. Gene ontology (GO) analysis of genes with hydroxymethylated promoter in $\mathrm{T}$ cells showed a marked enrichment for biological processes related to cell death and $\mathrm{T}$ cell regulation $\left(p<10^{-6}\right)$, as well as pathways related to $\mathrm{T}$ cell receptor signaling $\left(p<10^{-4}\right)$.

Taken together, we found high $5 \mathrm{hmC}$ density in gene bodies and showed that $5 \mathrm{hmC}$ levels decrease during cell differentiation.

\section{$5 \mathrm{hmC}$ enrichment at gene bodies positively correlates with gene expression}

To assess the potential gene regulatory role of $5 \mathrm{hmC}$ during human hematopoiesis, we integrated $5 \mathrm{hmC}$ profiles with measurement of gene expression by RNA-seq and genome-wide mapping of histone modifications using publically available ChIP-seq data sets.

We characterized $5 \mathrm{hmC}$ distribution around annotated genes. Figure 2 shows the enrichment of $5 \mathrm{hmC}-1.5-\mathrm{kb}$ upstream of the transcription start site (TSS) to $+1.5-\mathrm{kb}$ downstream of the transcription termination site (TTS). We found that gene body $5 \mathrm{hmC}$ correlated positively with gene expression in all analyzed blood cell types (Fig. 2a-d). Specifically, when we categorized $5 \mathrm{hmC}$ enrichment 5-kb downstream the TSS, we confirmed that low expressed genes contained low $5 \mathrm{hmC}$ levels, while highly expressed genes showed significantly higher $5 \mathrm{hmC}$ levels (Fig. 2e-h). Interestingly, $5 \mathrm{hmC}$ decreases especially at TSS of highly expressed genes [indicated by the blue line in the $5 \mathrm{hmC}$ heatmap (Fig. 2a-d)].

It is well known that repression of gene expression by DNA methylation mainly occurs around the TSS [14]. We therefore characterized $5 \mathrm{hmC}$ distribution around

\footnotetext{
(See figure on next page)

Fig. $15 \mathrm{hmC}$ levels and distribution in hematopoietic cells. a Proportion of hydroxymethylated sites in the indicated cell types. The number of $5 \mathrm{hmC}$ sites for each cell type was obtained following RRHP data analysis; $n=2$ for hESC, CB-CD34+ cells and granulocytes; $n=1$ for CD4+ T cells, CD19+ B cells and CD14+ monocytes. The mean numbers \pm SEM are presented. b Quantification of $5 \mathrm{hmC}$ per $1 \times 10^{6}$ nucleotide DNA in hESC $(n=1)$, CB-CD34+ $(n=2), C D 4+$ T cells $(n=3), C D 19+B$ cells $(n=1), C D 14+$ monocytes $(n=3)$ and granulocytes $(n=3)$. Cells were harvested, DNA isolated, hydrolyzed and analyzed for $5 \mathrm{hmC}$ using liquid chromatography tandem mass spectrometry (LC/MS/MS). 5hmC levels are normalized relative to $10^{6}$ nucleosides. Data shown are the mean \pm SEM. c Percentage of $5 \mathrm{hmC}$ in annotated genomic elements for each indicated cell type. Promoters were defined -1-kb upstream to +100-bp downstream of the transcription start site (TSS); transcription termination site (TTS) indicates a region -100-bp upstream to +1-kb downstream of the TTS. UTR untranslated region. $\mathbf{d}$ Relative density of $5 \mathrm{hmC}$ in annotated genomic elements for each indicated cell type. The relative density of $5 \mathrm{hmC}$ reflects the number of $5 \mathrm{hmC}$ encompassed in annotated genomic elements normalized to $10^{5}$ base pair of the respective element and to a fixed number of $10^{5} 5 \mathrm{hmC}$ sites
} 

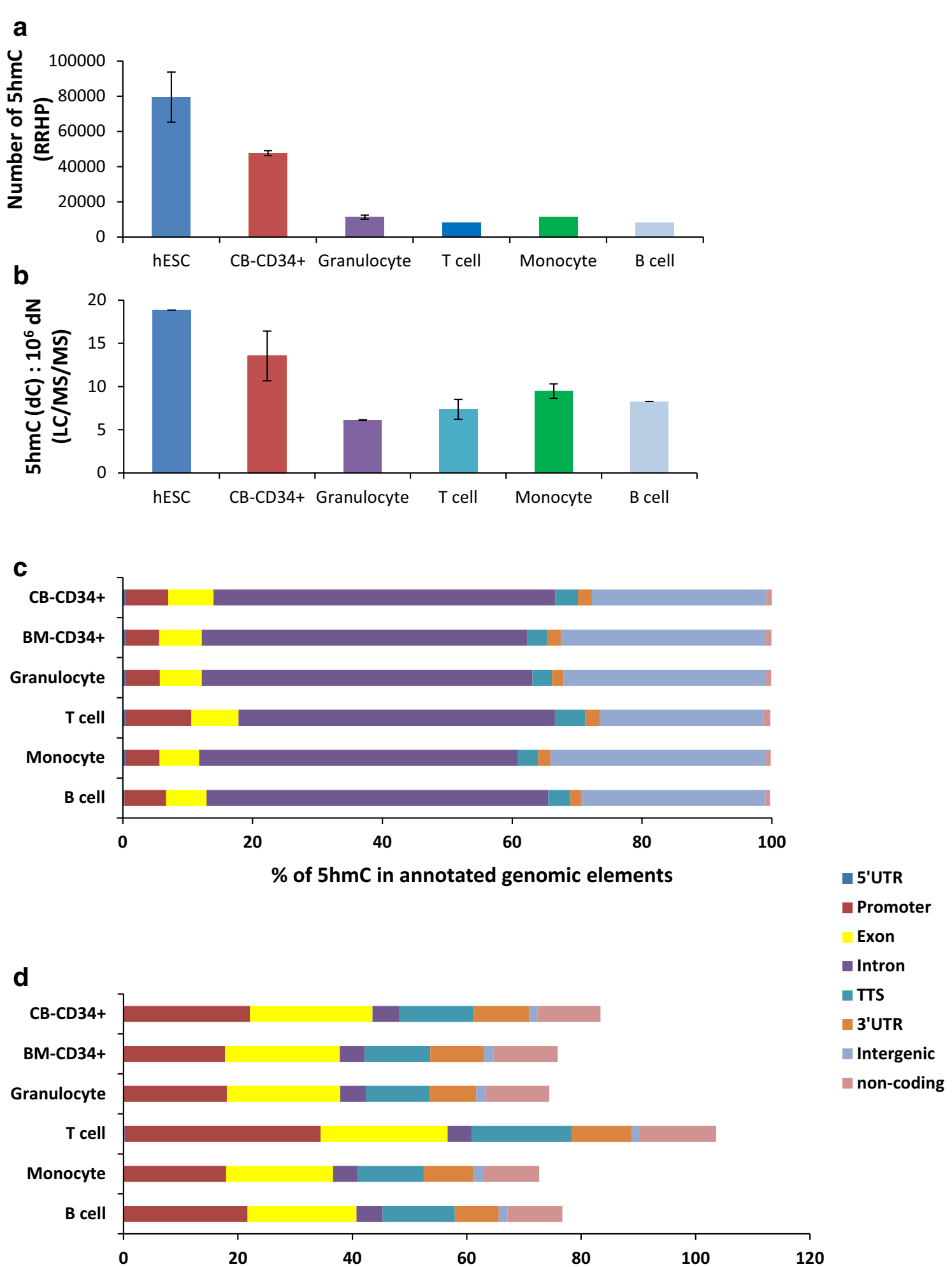

d

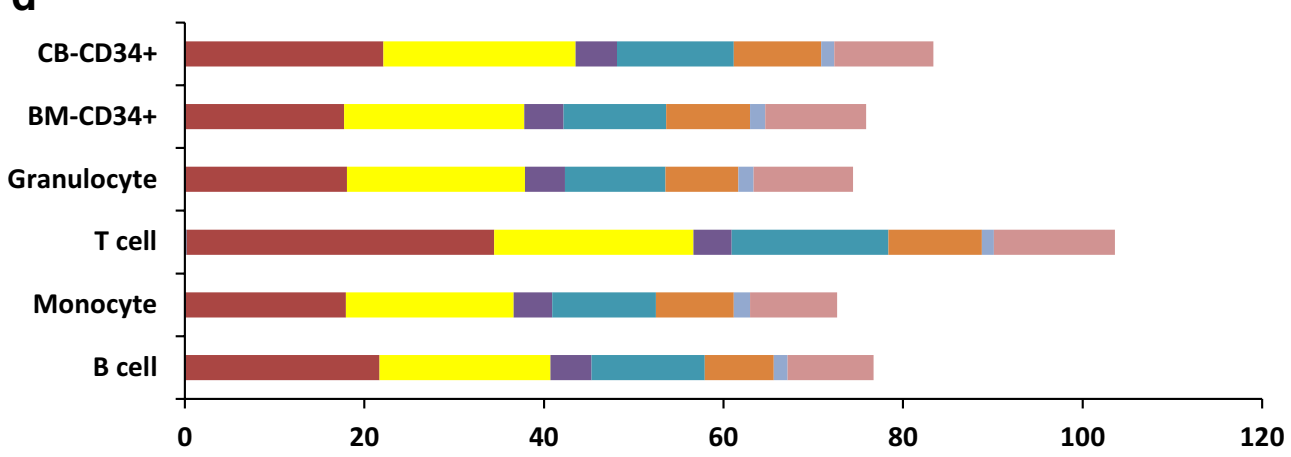

Relative density of $5 \mathrm{hmC}$ in annotated genomic elements 
a

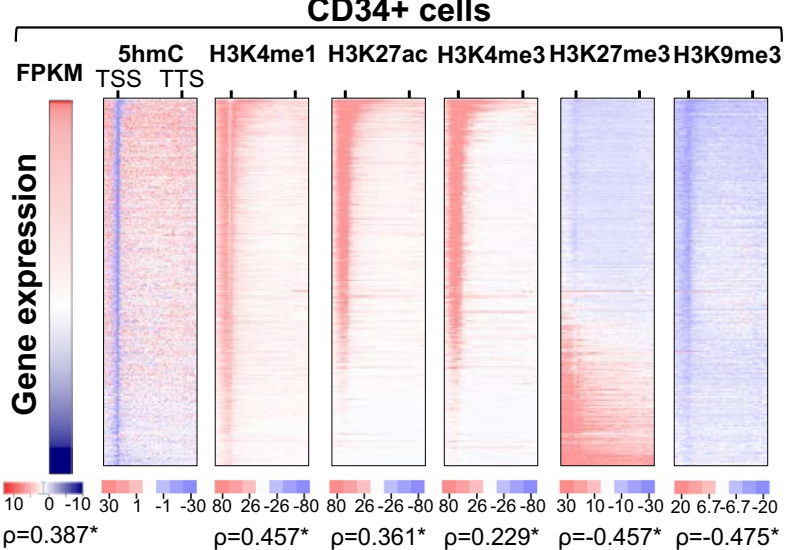

b

T cells

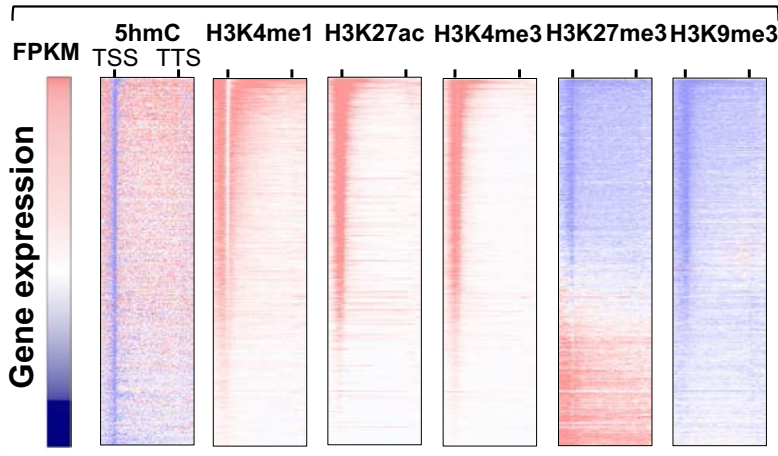

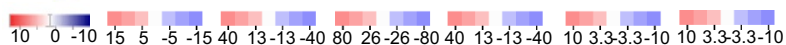
$\rho=0.437^{\star}$ $\rho=0.530^{*} \rho=0.377^{*} \rho=0.384^{*} \rho=-0.273 \rho=-0.355^{*}$
C

Monocytes

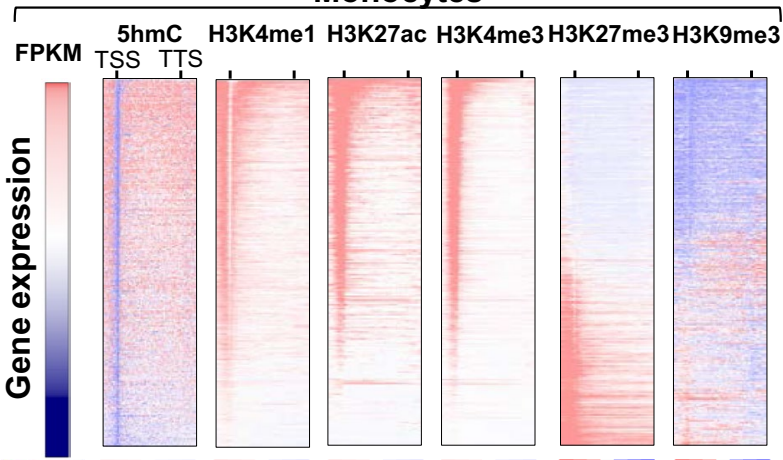

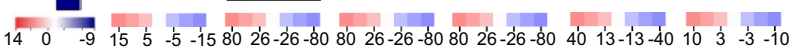
$\rho=0.394^{*}$ $\rho=0.479^{*} \quad \rho=0.478^{*} \quad \rho=0.340^{*} \quad \rho=-0.268 \quad \rho=-0.117$

d

\section{B cells}

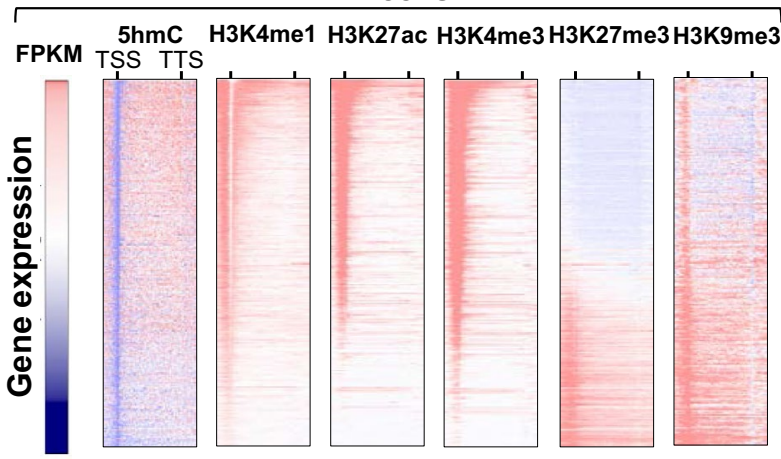

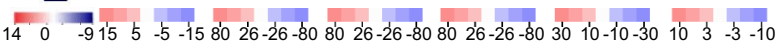
$\rho=0.431^{*} \quad \rho=0.566^{*} \quad \rho=0.433^{*} \quad \rho=0.338^{*} \quad \rho=-0.428^{*} \quad \rho=-0.274$ e

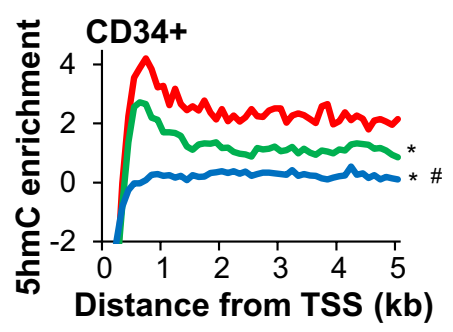

f

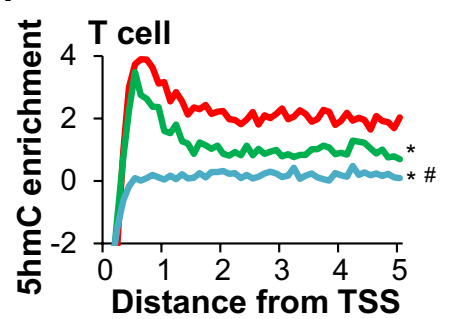

(kb) g

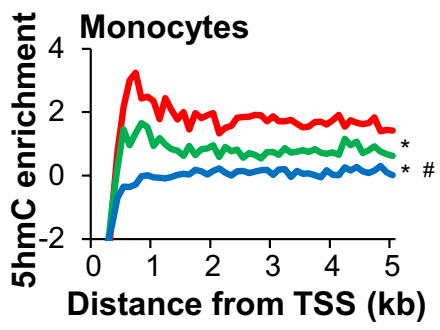

h

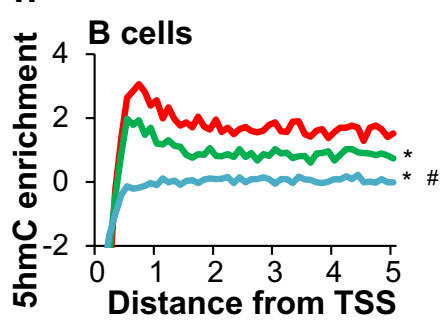

(kb)
- High gene expression

- Intermediate expression

— Low gene expression 
(see figure on previous page.)

Fig. $25 \mathrm{hmC}$ enrichment in gene bodies positively correlates with gene expression and active chromatin. Heatmaps showing the enrichment of the indicated epigenetic mark -1.5 -kb upstream of the TSS to $+1.5-\mathrm{kb}$ downstream of the TTS in all annotated human genes ordered by decreasing expression (log2-transformed FPKM values) in CD34+ (a), T cells (b), monocytes (c) and B cells $(\mathbf{d})$. The Spearman rank $(\rho)$ correlation coefficient below the heatmaps indicates the correlation between gene body $5 \mathrm{hmC}$ levels and gene expression (log2 FPKM) or a specific histone modification (exact permutation test for testing the null hypothesis of no correlation, two-tailed, ${ }^{*} p<5 \times 10^{-20}$ ). Averaged $5 \mathrm{hmC}$ enrichment in CD34+ (e), T cells (f), monocytes (g) and B cells (h) 5-kb downstream of the TSS of genes expressed at high, low or intermediate level. Genes were divided into tertiles based on expression levels and averaged $5 \mathrm{hmC}$ enrichment 5 - $\mathrm{kb}$ downstream of the TSS. Differences in $5 \mathrm{hmC}$ enrichment between the tertiles were assessed using the Kolmogorov-Smirnov test (*; ${ }^{\#}$ significant differences in $5 \mathrm{hmC}$ enrichment with $D>0.45$ and $p<10^{-10}$. ${ }^{*}$ High versus intermediate or low gene expression; "intermediate versus low gene expression)

TSS of annotated genes (Additional file 2: Fig. S2a-d) and found that the TSS of high expressed genes displayed higher levels of $5 \mathrm{hmC}$ than low expressed genes. When we increased the resolution of our analysis around the TSS $( \pm 5 \mathrm{~kb})$, by considering the $5 \mathrm{hmC}$ signal every 50 bp (Additional file 2: Fig. S2e-h), we found that low expressed genes displayed a modest increase in $5 \mathrm{hmC}$ signal around the TSS, while highly expressed genes showed an overall higher signal characterized by a marked 150 bp deep around the TSS, surrounded by two 350-bp peaks (Additional file 2: Fig. S2e-h).

Enrichment of $5 \mathrm{hmC}$ in the body of highly expressed genes also correlated with active chromatin regions marked by H3K4me1, H3K27ac and H3K4me3 (Fig. 2a-d). In contrast, we observed no or an inverse correlation between $5 \mathrm{hmC}$ enrichment and regions containing repressive histone marks H3K27me3 and H3K9me3 (Fig. 2a-d). In all analyzed blood cell types, up to 40 and $25 \%$ of the $5 \mathrm{hmC}$ were found within ChIP-seq peaks of active histone marks putative of poised enhancers H3K4me1 (40\%) or active enhancers H3K27ac (25\%) [15], while $<7 \%$ of $5 \mathrm{hmC}$ sites overlapped with repressive histone marks H3K27me3 and H3K9me3 (Additional file 2: Fig. S3). To rule out that the association of $5 \mathrm{hmC}$ with "active" histone modifications was attributed to an over-representation of CCGG sites within "active" histone ChIP-seq peaks, we compared the enrichment of $5 \mathrm{hmC}-\mathrm{CCGG}$ to unmodified-CCGG and confirmed significant $5 \mathrm{hmC}$ enrichment in the vicinity of active histone marks (Additional file 2: Fig. S3). These results demonstrate that genes enriched with $5 \mathrm{hmC}$ are in an active chromatin state.

\section{In CD34+ cells, $5 \mathrm{hmC}$ associates with transcription factor} binding at enhancers

TF binding is central in the regulation of gene expression during hematopoiesis and is known to be affected by epigenetic modifications such as DNA methylation [16]. We therefore used publically available ChIP-seq data sets [17] and investigated whether the presence of $5 \mathrm{hmC}$ at gene regulatory regions could affect binding of FLI1, RUNX1, GATA2 and ERG, TFs crucial for blood development.
We found that all four TFs bound chromatin enriched with H3K4me1, H3K27ac and H3K4me3 (Fig. 3; Additional file 2: Fig. S4), while no TF binding was detected in chromatin marked with H3K27me3 or H3K9me3 (Additional file 2: Fig. S4). At putative poised enhancers (H3K4me1 peaks), active enhancers (H3K27ac peaks or dually marked $\mathrm{H} 3 \mathrm{~K} 27 \mathrm{ac} / \mathrm{H} 3 \mathrm{~K} 4 \mathrm{me} 1$ regions), as well as predicted enhancers using the Hidden Markov Model of Ernst and Kellis: ChromHMM [18], RUNX1 and FLI1 binding was enhanced in the presence of $5 \mathrm{hmC}$ (Fig. 3a$\mathrm{h})$. The presence of $5 \mathrm{hmC}$ did not influence GATA2 and ERG binding (Fig. 3i-p).

RRHP allows us to assess $5 \mathrm{hmC}$ distribution in a CCGG-specific context [11]. Since TF binding is sequence specific, we ensured that increased binding of FLI1 and RUNX1 at enhancers positive for $5 \mathrm{hmC}$ was not biased toward the CCGG sequence. We therefore compared TF binding at histone modification peaks positive for CCGG and $5 \mathrm{hmC}$ versus histone peaks positive for CCGG but negative for $5 \mathrm{hmC}$ and confirmed a significantly higher binding of FLI1 and RUNX1 at active enhancers enriched with $5 \mathrm{hmC}$ (Additional file 2: Fig. S5). Taken together, these results show that the presence of $5 \mathrm{hmC}$ in regulatory regions may increase the recruitment of key hematopoietic TFs such as RUNX1 and FLI1 at enhancers.

$5 \mathrm{hmC}$ marks genes linked to hematopoietic differentiation To investigate whether $5 \mathrm{hmC}$ marks genes linked to hematopoiesis in a cell-type-specific manner, we performed unsupervised clustering based on gene body $5 \mathrm{hmC}$ levels for all annotated genes. We identified 3208 genes, which contained high gene body $5 \mathrm{hmC}$ levels in all analyzed hematopoietic cell types (Additional file 2: Fig. S6a; cluster E). Gene set enrichment analysis using GO and KEGG confirmed that these genes strongly associated with blood cell function (Additional file 2: Fig. S6b). A second unsupervised cluster analysis subdivided the 3208 genes into six clusters (Fig. 4a; Additional file 3: Table S2), allowing us to identify groups of genes with differential gene body $5 \mathrm{hmC}$ levels across the mature blood cell types and pointing to specific $5 \mathrm{hmC}$ profiles 


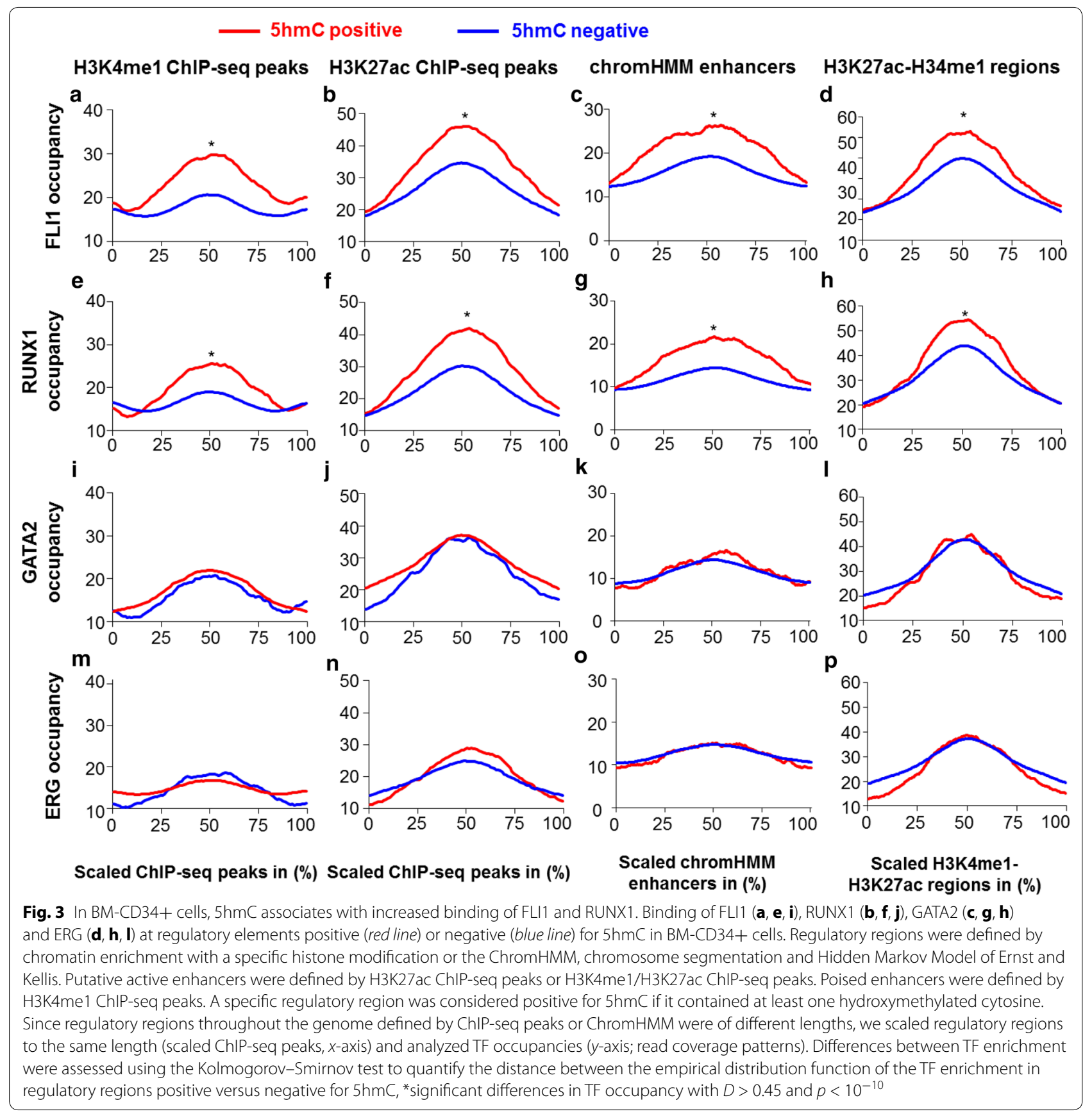

(See figure on next page)

Fig. 4 hmC marks genes important for blood cell type function. a Using Euclidian cluster calling and the complete linkage method, $3208 \mathrm{genes}$ (from cluster E in Additional file 2: Figure S4) were clustered according to their gene body $5 \mathrm{hmC}$ levels. The mean levels of gene body $5 \mathrm{hmC}$ from two biological replicates were used for granulocytes and CB-CD34+ cells. b Log2 of FPKM-averaged gene expression of all genes in the different clusters across the cell types. Only genes with FPKM $>1$ were included. Averaged log2 (FPKM) is shown $\pm 95 \%$ Cl. *Two-tailed unpaired Student's $t$ test; $p<0.001$. c Gene ontology (GO) and Kyoto Encyclopedia of Genes and Genomes (KEGG) analyses of genes falling in the six clusters. Only the first two GO and KEGG terms are listed. Heat density plots showing gene-wise changes in gene expression (FPKM, $y$-axis) versus changes in gene body 5hmC (x-axis) between progenitor BM-CD34+ cells and mature blood cells: T cells $(\mathbf{d}), B$ cells $(\mathbf{e})$ and monocytes (f). Only genes with FPKM and gene body $5 \mathrm{hmC}>1$ were plotted. The logarithmic fold change in gene expression needed to be $\geq 2$ to define increase or loss of gene expression in mature blood cells. In each plot, the Spearman rank correlation coefficient $\rho$ and the number $(n)$ of genes plotted are shown (exact permutation test for testing the null hypothesis of no correlation, two-tailed, $p<5 \times 10^{-20}$ ) 


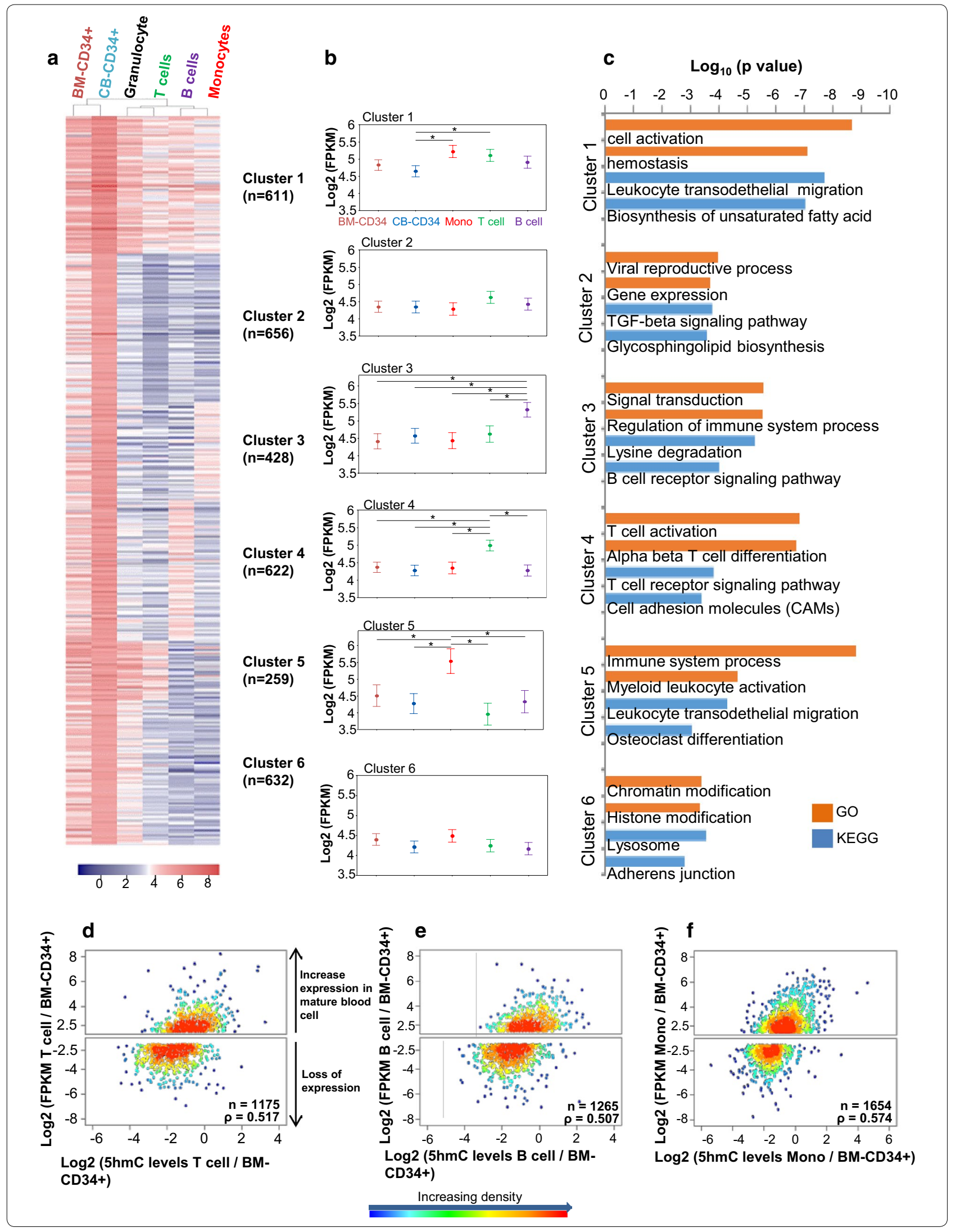


associated with definite blood cell function. Averaged expression of genes found in each cluster indicated that genes maintaining high gene body $5 \mathrm{hmC}$ levels during differentiation of CD34+ cells into a specific mature blood cell type were highly expressed in the respective blood cell type (Fig. 4b) and recapitulated mature blood cell function (GO and KEGG analysis; Fig. 4c).

Such specific changes of intragenic $5 \mathrm{hmC}$ levels during CD34+ cell differentiation into a mature blood cell type led us to investigate how changes in gene body $5 \mathrm{hmC} \mathrm{lev-}$ els correlated with changes in gene expression. We found that an increase in gene expression during differentiation of BM-CD34+ cells into a mature cell type associated with the preservation or gain of gene body $5 \mathrm{hmC}$ levels, while loss of gene expression in the committed cells correlated with a significant decrease in gene body $5 \mathrm{hmC}$ levels (Fig. 4d-f). Similar results were obtained when CB-CD34+ cells were used as reference cell type (Additional file 2: Fig. S7). Taken together, our results suggest that in CD34+ cells, $5 \mathrm{hmC}$ marks key genes required for lineage specification and mature blood cell function.

\section{Concomitant high gene body $5 \mathrm{hmC}$ levels and promoter DNA methylation prime genes for expression or repression during CD34+ cell differentiation}

Our data indicated that in CD34+ cells, $5 \mathrm{hmC}$ primes genes for further expression in differentiated cells. To validate dynamic changes of cytosine modifications associated with the regulation of gene expression during hematopoiesis, key genes were selected from the clusters obtained in Fig. 4a. TNFRSF25 is a marker of activated $\mathrm{T}$ cells, CD19 a surface marker of mature B cells, while FOXO1 is a TF important for lymphoid lineage commitment. AZU1 has been selected as a regulator of myeloid cell function and RUNX1 as a TF important for CD34+ cell homeostasis. We measured gene expression, promoter DNA methylation and integrated it with gene body $5 \mathrm{hmC}$ levels.

In $\mathrm{T}$ cells, $\mathrm{B}$ cells, monocytes and granulocytes, genes highly expressed contained significant levels of gene body $5 \mathrm{hmC}$ and low promoter DNA methylation. In contrast, high promoter DNA methylation and lack of gene body $5 \mathrm{hmC}$ were associated with low gene expression (Fig. 5). In CD34+ cells, DNA methylation of FOXO1, TNFRSF25, CD19 and AZU1 promoters was concomitant with high $5 \mathrm{hmC}$ gene body levels (Fig. 5ad). RUNX1, which is higher expressed in CD34+ cells, displayed lower promoter methylation in these cells (Fig. 5e). Our data indicate that in CD34+ cells, genes containing $5 \mathrm{hmC}$ and $5 \mathrm{mC}$ are primed for active transcription in mature blood cells. During differentiation, loss of gene body $5 \mathrm{hmC}$ will result in gene repression, while loss of promoter DNA methylation will allow gene expression.

\section{Discussion}

Here, we characterized for the first time the genomewide distribution of $5 \mathrm{hmC}$ in human CD34+ progenitor and mature blood lineage cells. Our main findings are that (1) the presence of $5 \mathrm{hmC}$ in gene bodies positively correlates with gene expression and active chromatin state, (2) in CD34+ cells, $5 \mathrm{hmC}$ primes the expression of genes that are important for myeloid and lymphoid cell differentiation, and (3) in CD34+ cells, the presence of $5 \mathrm{hmC}$ at enhancers may enhance binding of key hematopoietic TFs.

We showed that cells with higher renewal potential present higher levels of $5 \mathrm{hmC}$ compared to differentiated blood cells. Importantly, differentiated blood cell types maintained substantial levels of $5 \mathrm{hmC}$, suggesting a regulatory role for $5 \mathrm{hmC}$ rather than simply being an intermediate product during the demethylation process. Gene expression profiles of analyzed blood cells types revealed that highly expressed genes display higher levels of $5 \mathrm{hmC}$ in the immediate vicinity of TSS. This is in accordance with recent reports [10] and clearly demonstrates that $5 \mathrm{hmC}$ is found in active/open chromatin regions in hematopoietic cells.

Our study highlights the importance of dynamic changes of $5 \mathrm{hmC}$ distribution during CD34+ cell differentiation. A recent study by Madzo et al. [8] investigated the role of $5 \mathrm{hmC}$ during in vitro erythroid differentiation and also highlighted changes of $5 \mathrm{hmC}$ distribution during erythroid cell formation. Here, we used primary human cells to assess $5 \mathrm{hmC}$ function and distribution, which is of great importance since it has previously been shown that $5 \mathrm{hmC}$ levels decrease quickly during in vitro cell culture [19]. Two recent reports studied the role of $5 \mathrm{hmC}$ during mouse $\mathrm{T}$ and human $\mathrm{B}$ cell development into Th1/Th2 cells or plasma cells, respectively $[9,10]$. Importantly, our study included multipotent CD34+ cells and mature blood cell types, which are still poorly studied with respect to $5 \mathrm{hmC}$, and provides a valuable resource for a systematic analysis of $5 \mathrm{hmC}$ function during the whole hematopoietic differentiation process.

Several studies, including ours, link $5 \mathrm{hmC}$ to histone modifications indicative of enhancer [20]. In addition, we show that in CD34+ cells, the presence of $5 \mathrm{hmC}$ at putative active or poised enhancers associates with increased binding of RUNX1 and FLI1. Whether $5 \mathrm{hmC}$ is deposited passively in gene regulatory regions due to chromatin activity, or whether it functionally regulates $\mathrm{TF}$ binding and therefore gene expression remains incompletely understood. Madzo et al. [8] observed a dramatic 

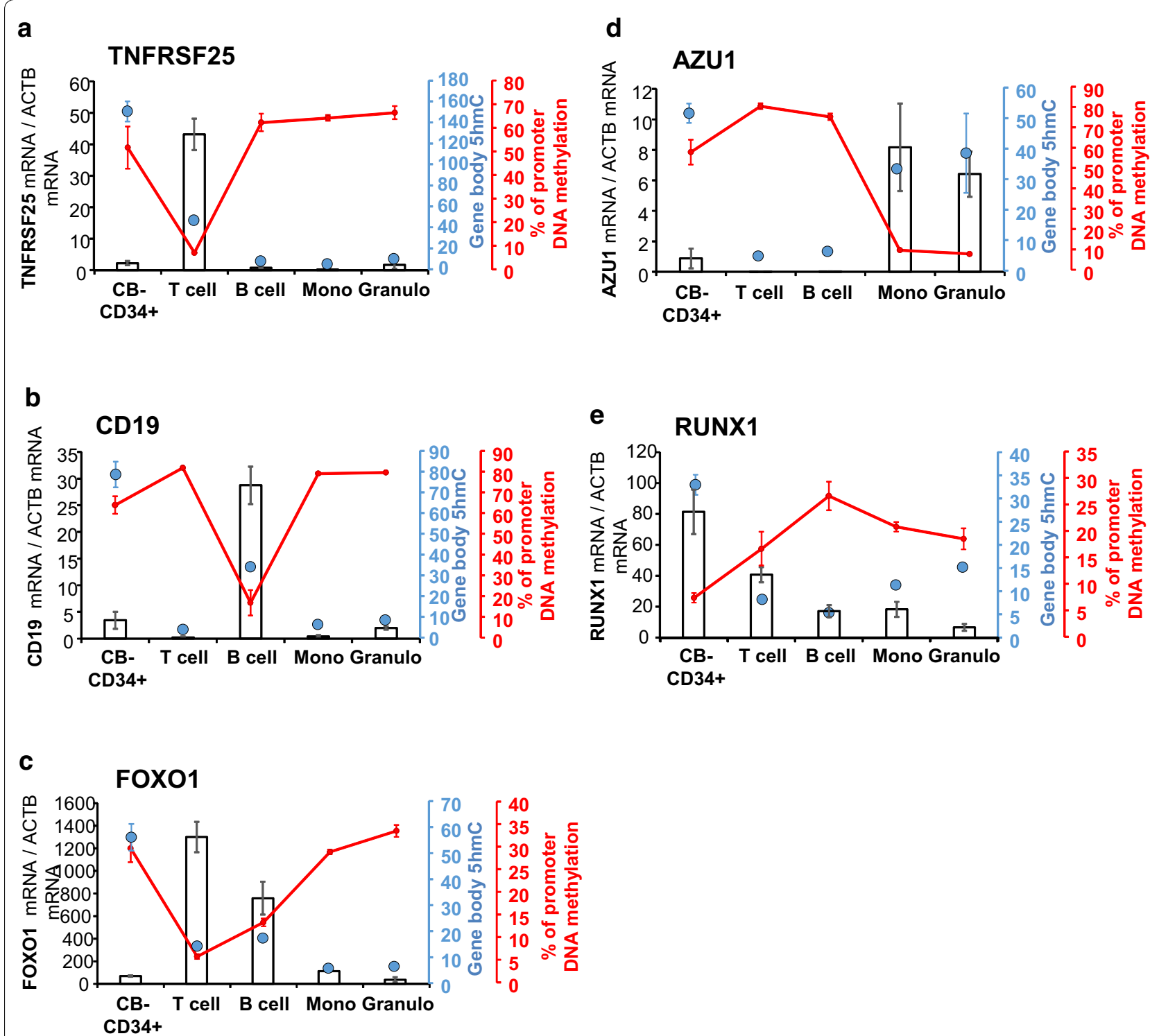

Fig. 5 Cytosine modification ( $5 \mathrm{hmC}, 5 \mathrm{mC}$ ) changes correlate with gene expression. Gene expression (bars), promoter DNA methylation (red line) and 5hmC gene body levels (blue dots) were assessed for CD19 (a), TNFRSF25 (b), FOXO1 (c), AZU1 (d) and RUNX1 (e). Gene expression levels were measured by real-time quantitative reverse transcription PCR and normalized to beta-actin levels. Promoter DNA methylation was assessed by bisulfite conversion and pyrosequencing. The percentage of methylation represents the average methylation of $\mathrm{CpG}$ sites in the region, and gene body $5 \mathrm{hmC}$ levels were measured by RRHP

decrease of $5 \mathrm{hmC}$ levels during erythroid differentiation compared to CD34+ cells, which is in agreement with our results. Moreover, the authors observed a strong correlation between loci that gained $5 \mathrm{hmC}$ and binding of TF known to be important for erythropoiesis, supporting that $5 \mathrm{hmC}$ may be a positive regulator of TF function. In agreement with our study, these results highlight that $5 \mathrm{hmC}$ may play an important role in regulating TF binding affinity to their target binding sites or in priming chromatin-remodeling processes to allow TFs to exert their functions. Therefore, inactivation of TET2, which is known to affect blood stem/progenitor cell renewal and differentiation $[7,21]$, would lead to reduced $5 \mathrm{hmC}$ levels and may consequently lead to aberrant TF binding and impaired gene expression.

Recent studies suggest that $5 \mathrm{hmC}$ has a destabilizing effect on DNA structure [22, 23], while $5 \mathrm{mC}$ stabilizes chromatin [24]. In CD34+ cells, several genes showed 
concomitant promoter DNA methylation and high gene body $5 \mathrm{hmC}$ levels. This "bivalent" $5 \mathrm{mC} / 5 \mathrm{hmC}$ status may prime genes for further epigenetic regulation of their expression during hematopoietic development. Loss of gene body $5 \mathrm{hmC}$ would result in gene repression, while loss of promoter DNA methylation would allow expression. The coexistence of epigenetic marks with opposite effects on gene expression has previously been described for histone marks (H3K4me3 and H3K27me3). The presence of "bivalent histone domains" maps key developmental genes that need to be turned on or off during differentiation [25]. Importantly, $5 \mathrm{hmC}$ was shown to associate with "bivalent histone domains" in ESC [26]. Our analysis suggests that in CD34+ cells, gene body $5 \mathrm{hmC}$ marks genes whose expression can be epigenetically regulated during differentiation into a specific mature blood cell type. Taking our results in perspective to those of others, we find that in multipotent CD34+ cells, $5 \mathrm{hmC}$ maps genes important for lineage specification and suggest that $5 \mathrm{hmC}$ may directly influence cell fate decisions.

\section{Conclusions}

We used a single-base resolution method to show that during human hematopoiesis, $5 \mathrm{hmC}$ accompanies extensive chromatin reprogramming, including TF binding, and marks cell-specific genes actively transcribed in differentiated cells that are known to be important for myeloid and lymphoid lineage commitment.

\section{Methods}

\section{Human blood cells}

Umbilical cord blood was collected by sterile procedure from healthy pregnancies. Peripheral blood was obtained from adult healthy donors. All subjects provided written informed consent. Blood cells were separated by density gradient, followed by cell-type-specific isolation using MACS MicroBeads (Miltenyi, Biotec). Bone marrow CD34+ cells, from a unique donor, were purchased from StemCell Technologies. Granulocytes, CD4+, CD19+, CD14+ and BM-CD34+ cells were obtained from males and females between 30 and 50 years old. All experiments were approved by the Regional Committee for Medical and Health Research Ethics (2012/588/REK and 2012/1969/REK).

\section{Reduced representation of hydroxymethylcytosine profiling (RRHP)}

RRHP was performed as previously described [11] by Zymo Research on service basis (Zymo Research, California, USA). In brief, genomic DNA was fragmented using MspI, the sticky ends obtained were reconstituted using specific adapters, $5 \mathrm{hmC}$ was then glucosylated, the modified fragments were submitted to a second MspI digestion, and the CCGG sites with a glucosylated $5 \mathrm{hmC}$ were protected from digestion and further amplified and sequenced using next-generation sequencing. For sequencing, equal volumes of each amplified library were pooled and diluted to $8 \mathrm{pM}$. Samples were sixplexed into a single 50-bp sequencing using an Illumina HiSeq. FASTQ files were aligned to the human reference genome (hg19) with Bowtie 0.12.8 using default parameters and best. Only mapped sequencing reads beginning with a CCGG tag were considered in the analysis. The number of sequencing reads at a CCGG site was considered to be proportional to the level of hydroxymethylation. To compare positive $5 \mathrm{hmC}$ among the different cell types we used a more stringent read cutoff than in Petterson et al. [11]. A CCGG site was considered hydroxymethylated if the number of sequencing reads at this site was $>101.8$ reads, which is the mean read count + SEM for all CCGG sites in hESC (the cell type with highest $5 \mathrm{hmC}$ levels). Information regarding the RRHP data for each sample and replicates can be found in Additional file 1: Table S1.

To compare the positive $5 \mathrm{hmC}$ within the different cell types we use a more stringent read cutoff than the original method paper [11]. Based on our more stringent selection of $5 \mathrm{hmC}$ sites we found a strong correlation in replicated cell types (hESC, CB-CD34+ and granulocytes) with Pearson's correlation $r>0.75$ and $p<0.05$ (Additional file 1: Table S1).

Gene body $5 \mathrm{hmC}$ levels were determined by dividing the number of sequencing read at CCGG sites by the number of CCGG sites in the gene body.

\section{DNA isolation and LC/MS/MS analysis}

DNA from hESC $(n=1)$, CB-CD34+ $(n=2)$, as well as CD4+ T cells $(n=3)$, CD19+ B cells $(n=1)$, CD14+ monocytes $(n=3)$ and granulocytes $(n=3)$, was isolated after proteinase K (Sigma, St. Louis, MO, USA) and RNase A digestion (Qiagen, Germany) followed by phenol-chloroform extraction and ethanol precipitation. For LC/MS/MS, DNA was hydrolyzed to nucleosides by incubation with nuclease $\mathrm{P} 1$, snake venom phosphodiesterase and alkaline phosphatase (Sigma-Aldrich, St. Louis, MO, USA). Three volumes of methanol were added after digestion, and the reactions were centrifuged at $16,000 \mathrm{~g}$ for $30 \mathrm{~min}$. The supernatants were dried under vacuum, and resulting residues were dissolved in $50 \mu \mathrm{l} 5 \%(\mathrm{v} / \mathrm{v})$ methanol for analysis. Chromatographic separation of nucleosides was performed using a Shimadzu Prominence HPLC system with a Ascentis Express C18 $150 \times 2.1 \mathrm{~mm}$ i.d. $(2.7 \mu \mathrm{m})$ reverse-phase column equipped with an 
Ascentis Express C18 $12.5 \times 2.1 \mathrm{~mm}$ i.d. $(2.7 \mu \mathrm{m})$ guard column (Agilent Technologies, USA), with a flow rate of $0.2 \mathrm{ml} / \mathrm{min}$ at ambient temperature. The mobile phase consisted of A ( $0.1 \%$ formic acid in water) and B ( $0.1 \%$ formic acid in methanol), starting with $95 \% \mathrm{~A} / 5 \% \mathrm{~B}$ for $0.5 \mathrm{~min}$, followed by a 6.5 -min linear gradient of $5-50 \%$ B, 2 min with $50 \% \mathrm{~B}$ and 6-min re-equilibration with the initial mobile phase conditions. Online mass spectrometry detection was performed using an $A B$ Sciex 5000 triple quadrupole mass spectrometer ( $A B$ Sciex, USA) with TurboionSpray probe operating in positive electrospray ionization mode. The deoxyribonucleosides were monitored by multiple reaction using the mass transitions $228.1 \rightarrow 112.1(\mathrm{dC}), 242.1 \rightarrow 126.1$ [5-me $(\mathrm{dC})]$ and $258.1 \rightarrow 142.1[5-\mathrm{hm}(\mathrm{dC})]$.

\section{RNA sequencing}

RNA from $\mathrm{CB}-\mathrm{CD} 34+, \mathrm{CD} 4+\mathrm{T}$ cells, CD14+ monocytes and CD19+ B cells was purified using the RNeasy Mini Kit (Qiagen). Samples with an RNA integrity number value $>8$ were used for sequencing. Libraries were prepared using TruSeq RNA Sample Preparation Kit v2 (Illumina) following the manufacturer's instructions and sequenced with 100-bp paired-end reads on a HiSeq2000 Illumina. RNA-Seq data sets of BM-CD34+ cells (GSE63569) were downloaded from the gene expression database of Gene Expression Omnibus (GEO) [27]. Reads were aligned to the human reference genome (hg19) by TopHat v2.0.13 [28] and assembled by cufflinks v2.1.1 [28] using default parameters. Quantification of gene expression was performed using cufflinks v2.1.1 with default parameters to obtain fragment per kilobase million (FPKM) value.

\section{Bisulfite conversion and pyrosequencing}

DNA bisulfite conversion and pyrosequencing were performed as previously described [29]. Primers used for pyrosequencing, target sequences analyzed and number of CpGs assessed are listed in Additional file 4: Table S3.

\section{Analysis of publically available chromatin immunoprecipitation sequencing (ChIP-seq)}

Histone modification and TF-ChIP-seq data sets were publicly available. The accession numbers and description of histone modification ChIP-seq data sets are listed in Additional file 5: Table S4. FLI1, RUNX1, GATA2 and ERG TF-ChIP-seq data sets [17] were downloaded from the gene expression database of GEO (GSE45144). Raw reads were aligned to the human reference genome (hg19) using Novoalign v2.08.02 (http://www.novocraft. $\mathrm{com} /$ products/novoalign/) and default parameters. Reads with low quality $\leq 20$ were removed. Chromatin enriched with a specific histone modification or TF binding was identified using MACS v1.4.1 with default parameters and respective input samples as background.

\section{Prediction of active and poised enhancers}

Using histone modification ChIP-seq peaks we defined active enhancers as regions enriched with H3K27ac, while putative poised enhancers were regions enriched with H3K4me1. We also used regions enriched with both histone marks (H3K27ac and H3K4me1); here, an H3K27ac peak was considered to be positive for H3K4me1, if at least $25 \%$ of the H3K27ac peak was covered by H3K4me1 peaks. The H3K27ac/H3K4me1 regions were defined as active enhancers if they lacked TSS (TSS $\pm 1000 \mathrm{bp}$ ). We also used the predicted enhancer region of CD34+ cells using the ChromHMM segmentation of Ernst and Kellis [18]. Predicted enhancer regions using the Hidden Markov Model were obtained from The NIH Roadmap Epigenomics Mapping Consortium.

\section{Quantitative RT-PCR}

cDNA synthesis and qRT-PCR were performed as previously described [29]. Expression levels of target genes were normalized to beta-actin expression. Primer sequences are listed in Additional file 6: Table S5.

\section{Statistical analyses}

Student's unpaired two-tailed t test was used to compare means. In case of non-normal distribution, the Mann-Whitney test was used to compare medians. $p$ value $<0.05$ was considered statistically significant. Hierarchical clustering was applied to $\log 2$ gene body $5 \mathrm{hmC}$ levels using the Euclidean distance and complete linkage method. We used HOMER to perform GO and KEGG functional enrichment analyses, based on all RefSeq genes as reference. The deepTools suite [30] and the computeMatrix command in scale-region mode were used to extract the scores of enrichment and produce the heatmaps of $5 \mathrm{hmC}$ and histone modification enrichments in gene bodies. $5 \mathrm{hmC}$ enrichment in gene bodies was obtained by normalizing RRHP-CCGG reads to distribution of CCGG sites in the human genome. Histone modification enrichment was obtained by normalizing ChIP-seq reads to input reads. The deepTools suite was used to analyze TFs occupancies ( $y$-axis) in regulatory regions (scaled ChIP-seq peaks, $x$-axis) by normalizing ChIP-seq reads for RUNX1, FLI1, GATA2 or ERG to input reads. Kolmogorov-Smirnov test was used to quantify the distance between the empirical distribution function of two conditions. 


\section{Availability of data and materials}

The data sets supporting the conclusions of this article are available in the NCBI GEO (http://www.ncbi.nlm.nih. gov/geo/) under accession number GSE69905.

\section{Additional files}

Additional file 1: Table S1. Summary of RRHP sequencing data. Table describes RRHP sequencing depth, percentage of aligned reads, raw and normalized $5 \mathrm{hmC}$ counts as well as correlation.

Additional file 2: Figure S1. Quantification of 5-methylcytosine using LC/MS/MS. Quantification of 5-methylcytosine $(5 \mathrm{mC})$ per $10^{6}$ unmodified bases in hESC $(n=1), C B-C D 34+(n=2), C D 4+T$ cells $(n=3)$, CD19+ B cells $(n=1), C D 14+$ monocytes $(n=3)$, and granulocytes $(n=3)$ assessed by LC/MS/MS. $n$ indicates the number of independent DNA measured for each cell type. Figure $\mathbf{S 2}$ : $5 \mathrm{hmC}$ at transcription start sites. Average enrichment of $5 \mathrm{hmC}$ RRHP reads (signal) along a $100 \mathrm{~kb}$ window $(\mathbf{a}-\mathbf{d})$ and $5 \mathrm{~kb}$ window $(\mathbf{e}-\mathbf{h})$ around the transcription start sites (TSS) of high and low expressed genes in the indicated hematopoietic cell types. Mean gene FPKM was used as threshold to dichotomize gene expression in high/low according to RNA-seq data in the same cell-types. Figure S3: $5 \mathrm{hmC}$ and histone modifications. Percentage of $5 \mathrm{hmC}$ or CCGG sites encompassed in ChIP-seq peaks of indicated histone modifications. The mean percentage of $5 \mathrm{hmC}$ in ChIP-seq peaks from two independent RRHP measurements in CB-CD34+ cells and one in $\mathrm{BM}-\mathrm{CD} 34+$ are shown \pm SEM. The mean percentages of CCGG sites found in ChIP-seq peaks of CD34, T cell, monocyte and B cell are shown. ${ }^{* * *}$ or * indicate significant difference between CCGG distribution in ChIP-seq peaks versus $5 \mathrm{hmC}$ distribution in ChIP-seq peaks of hematopoietic cells. ${ }_{* * *} p<0.0001,{ }^{*} p<0.05$; Student's unpaired two tailed t-test. Figure S4: $5 \mathrm{hmC}$ and transcription factor occupancy at inactive chromatin. FLI1, RUNX1, GATA2 and ERG occupancies at putative active promoters (H3K4me3 peaks), putative inactive chromatin enriched with H3K27me3 or H3K9me3. A specific ChIP-seq peak was considered positive for $5 \mathrm{hmC}$ when it contained at least one hydroxymethylated cytosine. In histone modification ChIP-seq-peaks positive or negative for $5 \mathrm{hmC}$ we assessed how TF-ChIP-seq reads were distributed which reflected the level of occupancy of an inactive chromatin region by a TF. The diagrams show the occupancy of a TF in inactive chromatin positive (red line) or negative (blue line) for $5 \mathrm{hmC}$. Figure $\mathbf{S 5}$ : Transcription factor occupancy at putative regulatory elements. FLI1, RUNX1, GATA2, ERG occupancies at regulatory elements in $\mathrm{BM}-\mathrm{CD} 34+$ cells. Regulatory regions were defined by chromatin enrichment with histone modifications: H3K4me1 or H3K27ac-ChIP-seq peaks defined putative active enhancers, H3K4me3ChIP-seq peaks defined active promoter regions. A specific regulatory region was considered positive for $5 \mathrm{hmC}$ if it contained at least one $5 \mathrm{hmC}$ site. In histone modification ChIP-seq-peaks we assessed how particular transcription factor ChIP-seq reads were distributed, which is proportional to binding. Occupancy of the indicated transcription factors in regulatory elements containing 5hmC-positive CCGG (red line) or 5hmC-negative CCGG (blue line) sites are shown. Enrichment of TFs (y-axis) at regulatory regions (scaled ChIP-seq peaks, x-axis). Differences between TF enrichment were assessed using the Kolmogorov-Smirnov test to quantify the distance between the empirical distribution function of the TF enrichment in regulatory regions positive versus negative for $5 \mathrm{hmC}, *$ indicates significant differences in TF occupancy with $D>0.3$ and $p<5.10^{-4}$. Figure S6: Changes in $5 \mathrm{hmC}$ gene body levels. a: Heat map representation of all known human genes clustered according to their gene body $5 \mathrm{hmC}$ levels. Euclidian cluster calling was used to produce the heat map. The five clusters identified are labeled with a letter and the number of genes per cluster is indicated. The mean levels of gene body $5 \mathrm{hmC}$ from two biological replicates were used for granulocytes and CB-CD34+ cells. b: Gene ontology (GO) and Kyoto Encyclopedia of Genes and Genomes (KEGG) analyses of genes identified in the five clusters in panel $\mathrm{A}$. The first two $\mathrm{GO}$ and KEGG terms are listed. Figure S7: Changes in $5 \mathrm{hmC}$ gene body levels versus changes in expression. Heat density plots depict the changes in gene expression (FPKM, $y$ axis) versus changes in gene body $5 \mathrm{hmC}(x$ axis) from progenitor cells (CB-CD34+ cells) to mature blood cells: T cells (a), B cells (b) and monocytes (c). Only genes with FPKM and gene body $5 \mathrm{hmC}>1$ were plotted. The logarithmic fold change in gene expression needed to be $>2$ to define gain or $<2$ to define loss of gene expression. In each plot, the Spearman rank correlation coefficient $\rho$ and the number (n) of genes plotted are shown (exact permutation test for testing the null hypothesis of no correlation, two tailed, $\mathrm{p}<5 \times 10^{-20}$ ).

Additional file 3: Table S2. Gene list described in Fig. 4a.

Additional file 4: Table S3. Primer used in pyrosequencing and coordinates of DNA sequence analyzed.

Additional file 5: Table S4. Publically available histone ChIP-seq data sets used.

Additional file 6: Table S5. Primers used in qRT-PCR.

\section{Abbreviations}

5mC: 5-methylcytosine; 5hmC: 5-hydroxymethylcytosine; BM: bone marrow; CB: cord blood; CD: cluster of differentiation; ERG: ETS-related gene; FLI1: friend leukemia integration 1; FPKM: fragment per kilobase million; GO: gene ontology; hESC: human embryonic stem cells; KEGG: Kyoto Encyclopedia of Genes and Genomes; RRHP: reduced representation of 5-hydroxymethylcytosine profiling; RUNX1: runt-related transcription factor 1; TET: ten-eleven-translocation; TF: transcription factor; TSS: transcription start site; TTS: transcription termination site.

\section{Authors' contributions}

XT and JS designed the original study. XT, CBV and MKK performed research. $X T, A U$ and $A H$ designed and performed bioinformatics analysis. ACS and ID provided essential research material. RL, IGM and AK analyzed data. XT, AU and IS analyzed data and drafted the manuscript. All authors read and approved the final manuscript.

\section{Author details}

${ }^{1}$ Nordic European Molecular Laboratory (EMBL) Partnership, Centre for Molecular Medicine Norway, University of Oslo, Blindern, P.O. Box 1137, 0318 Oslo, Norway. ${ }^{2}$ Department of Molecular Oncology, Institute for Cancer Research, Oslo University Hospital, Oslo, Norway. ${ }^{3}$ Department of Cancer Research and Molecular Medicine, Norwegian University of Science and Technology, Trondheim, Norway. ${ }^{4}$ Department of Medical Genetics, University of Oslo, Oslo University Hospital, Oslo, Norway. ${ }^{5}$ Department of Pharmacology, Oslo University Hospital, Ullevål, Oslo, Norway. ${ }^{6}$ Department of Obstetrics and Gynecology, Oslo University Hospital, University of Oslo, Oslo, Norway. ${ }^{7}$ Department of Haematology, Oslo University Hospital, Oslo, Norway. ${ }^{8}$ PCUK Movember Centre of Excellence, CCRCB, Queen's University, Belfast, UK. ${ }^{9}$ Clinic for Diagnostics and Intervention, Institute of Medical Microbiology, Oslo University Hospital, University of Oslo, Oslo, Norway. ${ }^{10}$ Norwegian Center for Stem Cell Research, University of Oslo, Oslo, Norway. ${ }^{11}$ Present Address: Department of Genetics, Institute for Cancer Research, Oslo University Hospital - The Norwegian Radium Hospital, Oslo, Norway.

\section{Acknowledgements}

JS is supported by the Norwegian Research Council, the Norwegian Cancer Society, South-East Norway Regional Health Authorities and the University of Oslo. We thank Lise Øhra Levy for collecting umbilical cord blood. The research leading to these results has received funding from the European Union Seventh Framework Programme (FP7-PEOPLE-2013-COFUND) under Grant Agreement No. 609020-Scientia Fellows. LC/MS/MS was performed by the Proteomics and Metabolomics Core Facility (PROMEC), Norwegian University of Science and Technology (NTNU), funded by the Faculty of Medicine at NTNU and Central Norway Regional Health Authority.

\section{Competing interests}

The authors declare that they have no competing interests.

Received: 28 February 2016 Accepted: 20 May 2016

Published online: 31 May 2016 


\section{References}

1. van Vlerken LE, Hurt EM, Hollingsworth RE. The role of epigenetic regulation in stem cell and cancer biology. J Mol Med. 2012;90:791-801.

2. Tahiliani M, Koh KP, Shen Y, Pastor WA, Bandukwala H, Brudno Y, et al. Conversion of 5-methylcytosine to 5-hydroxymethylcytosine in mammalian DNA by MLL partner TET1. Science. 2009;324:930-5.

3. Branco MR, Ficz G, Reik W. Uncovering the role of 5-hydroxymethylcytosine in the epigenome. Nat Rev Genet. 2012;13:7-13.

4. Ficz G, Branco MR, Seisenberger S, Santos F, Krueger F, Hore TA, et al. Dynamic regulation of 5-hydroxymethylcytosine in mouse ES cells and during differentiation. Nature. 2011:473:398-U589.

5. Ko M, Bandukwala HS, An J, Lamperti ED, Thompson EC, Hastie R, et al. Ten-eleven-translocation 2 (TET2) negatively regulates homeostasis and differentiation of hematopoietic stem cells in mice. Proc Natl Acad Sci USA. 2011;108:14566-71.

6. Quivoron C, Couronne L, DellaValle V, Lopez CK, Plo I, Wagner-Ballon O, et al. TET2 inactivation results in pleiotropic hematopoietic abnormalities in mouse and is a recurrent event during human lymphomagenesis. Cancer Cell. 2011;20:25-38.

7. Cimmino L, Abdel-Wahab O, Levine RL, Aifantis I. TET family proteins and their role in stem cell differentiation and transformation. Cell Stem Cell. 2011:9:193-204

8. Madzo J, Liu H, Rodriguez A, Vasanthakumar A, Sundaravel S, Caces $\mathrm{DBD}$, et al. Hydroxymethylation at gene regulatory regions directs stem/ early progenitor cell commitment during erythropoiesis. Cell Rep. 2014;6:231-44.

9. Caron G, Hussein M, Kulis M, Delaloy C, Chatonnet F, Pignarre A, et al. Cell-cycle-dependent reconfiguration of the DNA methylome during terminal differentiation of human B cells into plasma cells. Cell Rep. 2015;13:1059-71

10. Tsagaratou A, Aijo T, Lio CW, Yue X, Huang Y, Jacobsen SE, et al. Dissecting the dynamic changes of 5-hydroxymethylcytosine in T-cell development and differentiation. Proc Natl Acad Sci USA. 2014:111:E3306-15.

11. Petterson A, Chung TH, Tan D, Sun XG, Jia XY. RRHP: a tag-based approach for 5-hydroxymethylcytosine mapping at single-site resolution. Genome Biol. 2014;15(9):456.

12. Ruzov A, Tsenkina Y, Serio A, Dudnakova T, Fletcher J, Bai Y, et al. Lineagespecific distribution of high levels of genomic 5-hydroxymethylcytosine in mammalian development. Cell Res. 2011;21:1332-42.

13. Yu M, Hon GC, Szulwach KE, Song CX, Zhang L, Kim A, et al. Base-resolution analysis of 5-hydroxymethylcytosine in the mammalian genome. Cell. 2012;149:1368-80.

14. Jones PA. Functions of DNA methylation: islands, start sites, gene bodies and beyond. Nat Rev Genet. 2012:13:484-92.

15. Heintzman ND, Hon GC, Hawkins RD, Kheradpour P, Stark A, Harp LF, et al. Histone modifications at human enhancers reflect global cell-typespecific gene expression. Nature. 2009;459:108-12.
16. Medvedeva YA, Khamis AM, Kulakovskiy IV, Ba-Alawi W, Bhuyan MS, Kawaj $\mathrm{H}$, et al. Effects of cytosine methylation on transcription factor binding sites. BMC Genomics. 2014;15:119.

17. Beck D, Thoms JAl, Perera D, Schutte J, Unnikrishnan A, Knezevic K, et al. Genome-wide analysis of transcriptional regulators in human HSPCs reveals a densely interconnected network of coding and noncoding genes. Blood. 2013;122:E12-22.

18. Ernst J, Kellis M. Discovery and characterization of chromatin states for systematic annotation of the human genome. Nat Biotechnol. 2010;28:817-25.

19. Nestor CE, Ottaviano R, Reinhardt D, Cruickshanks HA, Mjoseng HK, McPherson RC, et al. Rapid reprogramming of epigenetic and transcriptional profiles in mammalian culture systems. Genome Biol. 2015;16(1):11

20. Stroud H, Feng SH, Kinney SM, Pradhan S, Jacobsen SE. 5-Hydroxymethylcytosine is associated with enhancers and gene bodies in human embryonic stem cells. Genome Biol. 2011;12(6):R54.

21. Moran-Crusio K, Reavie L, Shih A, Abdel-Wahab O, Ndiaye-Lobry D, Lobry

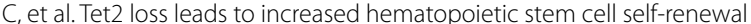
and myeloid transformation. Cancer Cell. 2011;20:11-24.

22. Wanunu M, Cohen-Karni D, Johnson RR, Fields L, Benner J, Peterman N, et al. Discrimination of methylcytosine from hydroxymethylcytosine in DNA molecules. J Am Chem Soc. 2011;133:486-92.

23. Lopez CMR, Lloyd AJ, Leonard K, Wilkinson MJ. Differential effect of three base modifications on DNA thermostability revealed by high resolution melting. Anal Chem. 2012;84:7336-42.

24. Jones PA, Laird PW. Cancer epigenetics comes of age. Nat Genet. 1999:21:163-7.

25. Bernstein BE, Mikkelsen TS, Xie XH, Kamal M, Huebert DJ, Cuff J, et al. A bivalent chromatin structure marks key developmental genes in embryonic stem cells. Cell. 2006;125:315-26.

26. Pastor WA, Pape UJ, Huang Y, Henderson HR, Lister R, Ko M, et al. Genome-wide mapping of 5-hydroxymethylcytosine in embryonic stem cells. Nature. 2011:473:394-7.

27. Dolatshad H, Pellagatti A, Fernandez-Mercado M, Yip BH, Malcovati L, Attwood $\mathrm{M}$, et al. Disruption of SF3B1 results in deregulated expression and splicing of key genes and pathways in myelodysplastic syndrome hematopoietic stem and progenitor cells. Leukemia. 2015;29:1092-103.

28. Trapnell C, Roberts A, Goff L, Pertea G, Kim D, Kelley DR, et al. Differential gene and transcript expression analysis of RNA-seq experiments with TopHat and Cufflinks. Nat Protoc. 2012;7:562-78.

29. Tekpli X, Zienolddiny S, Skaug V, Stangeland L, Haugen A, Mollerup S. DNA methylation of the CYP1A1 enhancer is associated with smoking-induced genetic alterations in human lung. Int J Cancer 2012;131:1509-16.

30. Ramirez F, Dundar F, Diehl S, Gruning BA, Manke T. deepTools: a flexible platform for exploring deep-sequencing data. Nucleic Acids Res. 2014:42:W187-91.

\section{Submit your next manuscript to BioMed Central and we will help you at every step:}

- We accept pre-submission inquiries

- Our selector tool helps you to find the most relevant journal

- We provide round the clock customer support

- Convenient online submission

- Thorough peer review

- Inclusion in PubMed and all major indexing services

- Maximum visibility for your research

Submit your manuscript at www.biomedcentral com/submit
C BioMed Central 